\title{
Optimal analysis of the cleaning process by ultrasonic system of vicuña wool
}

DOI: $10.46932 /$ sfjdv3n1-082

Received in: Jan 30st, 2021

Accepted in: Feb 1th, 2022

J. Alan Calderón Ch.

Pontificia Universidad Católica del Perú, Department of Mechanical Engineering, Perú. TU Ilmenau University of Technology, Applied Nanophysics, Institute for Physics, Germany E-mail: alan.calderon@pucp.edu.pe

\section{Benjamín Barriga}

Pontificia Universidad Católica del Perú, Department of Mechanical Engineering, Perú. E-mail: bbarrig@ pucp.edu.pe

Jorge Alencastre

Pontificia Universidad Católica del Perú, Department of Mechanical Engineering, Perú. E-mail: jalenca@pucp.edu.pe

\section{Julio C. Tafur}

Pontificia Universidad Católica del Perú, Department of Mechanical Engineering, Perú. E-mail: jtafur@pucp.edu.pe

\begin{abstract}
Alan Ccarita
Pontificia Universidad Católica del Perú, Department of Mechanical Engineering, Perú. E-mail: alan.ccarita@pucp.pe

\section{Dustin Rojas}

Pontificia Universidad Católica del Perú, Department of Mechanical Engineering, Perú.
\end{abstract}

\author{
A.M. Gómez-Amador \\ Universidad Carlos III de Madrid, España. \\ E-mail: amgomez@ing.uc3m.es
}

\begin{abstract}
This article has presented the techniques and strategies that were used to achieve the cleaning of vicuña wool by optimal analysis of ultrasonic systems. Usually, artisanal workers do it manually with some chemical components. However, they do not achieve a professional cleaning and damage the wool. Moreover with an increase in electricity consumption due to the fact that the washing is carried out on average at temperatures close to $50^{\circ} \mathrm{C}$. This was the case of this article to solve the problem through an optimal analysis that is suggested to be used in the designed washing machine that uses ultrasound. By a system identification suitable for the elaborated washing machine, the frequency necessary for the optimal cleaning is adjusted as the consequence of the polynomial analysis that was made offline. Finally, in this article the cleaning the vicuña wool is verified by a microscopic scale analysis, owing to the tests are presented photographically and they were compared through the three-dimensional reconstruction technique that was used to improve the results, which validate the cleaning of the vicuña wool.
\end{abstract}


Keywords: Ultrasound, system identification, control.

\section{INTRODUCTION}

The industry of washing machines is proposed to be enhanced by ultrasound, such as it is shown in the figure 1 , which is a portable ultrasonic cleaner.

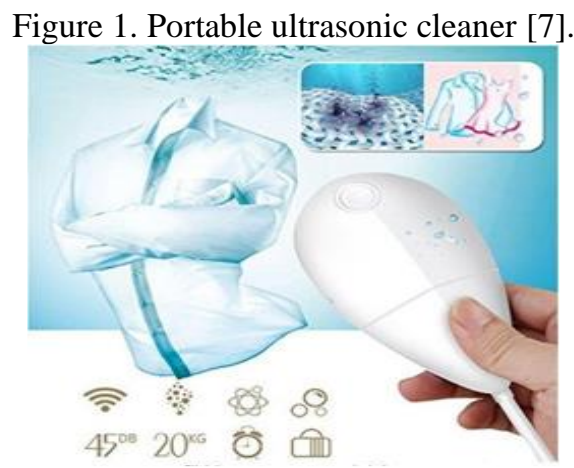

This portable device is quite useful to clean different types of fibres or wool, furthermore, this is a product in high demand. Nevertheless, it has a disadvantage due to the portable ultrasound cleaner is not usual to achieve a controlled and variable frequency. Therefore, it can not be used for washing delicate fibres, such as vicuña wool.

The vicuña is the native Andes animal of South America, which is showed in the figure 2. The vicuña wool is very tiny (12 um in average diameter) and highly valued by the textile industry. The optimization of ultrasound washing machine system will allow agricultural communities in Peru to make business by the cleaned vicuña wool and it will provide an incoming source, while communities are agree to use this machine according to care their traditions and environment conditions.

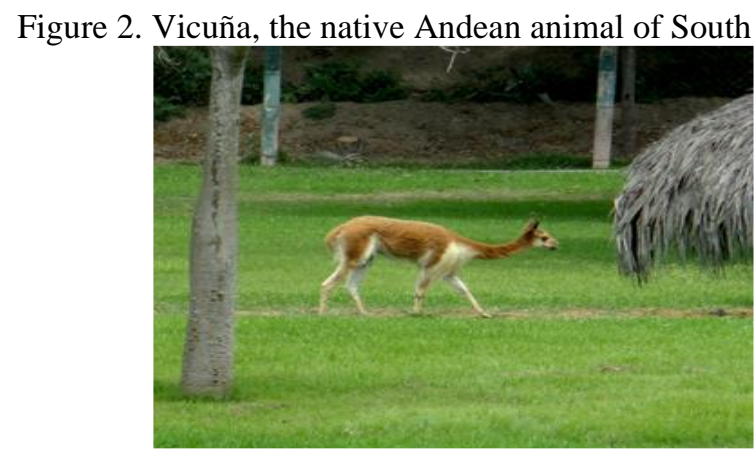

The price of vicuña wool is more expensive than $\$ 400 / \mathrm{kg}$ even though it is not cleaned, when it is just taken from vicuñas. Nevertheless, the alpaca wool was used in the experiments, because it has a similar diameter and no complications to find it in the market. After to make some experiments with 
alpaca wool, it will be extrapolated and contrasted with vicuña wool by the achieved results of the next research. The alpaca wool is shown in the figure 3.

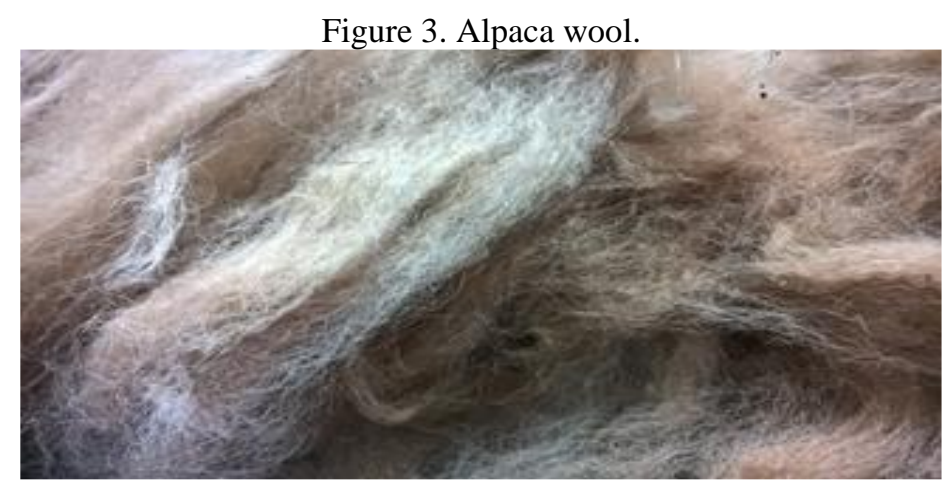

The optimization model prepared in this research and it helped to design the optimization algorithm that was based on the polynomial analysis, due to finding the frequency range of work according to wash the wool, but for this research this task was obtained offline (the consequent research will show the online analysis).

Furthermore, it was implemented a 3D reconstruction algorithm owing to evaluate the cleaning performance of the alpaca wool.

\section{EQUIPMENT}

In the following paragraphs are described the devices and equipment, which were used to make the experiments.

\subsection{VIBRATION GENERATOR}

The vibration generator based on piezoactuators with the capacity to adjust the frequency under offline operation, which was used due to produce vibration in the water of the washing machine [1].

This washing machine actuators (in which was evaluated the algorithm and the designed sensor in this article) were provided by the responsible person and designer of the ultrasound washing machine project, the engineer Benjamín Barriga, who also is one co-authors of this article. Therefore, the proposed optimization in this research is warranted by a vibration source that was adjusted from $0 \mathrm{~Hz}$ to $45 \mathrm{kHz}$ and which is shown in the figure 4 . 
Figure 4. Ultrasound actuator [1].

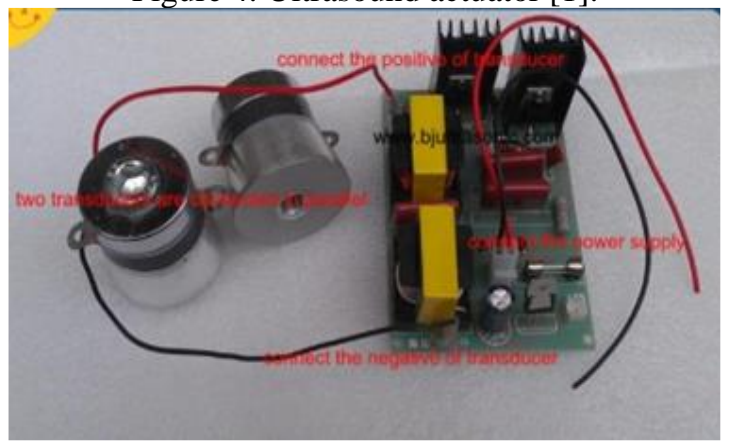

\subsection{VIBRATION SENSOR}

In order to measure the propagated vibration over the water to wash the alpaca wool, it was used a vibration sensor, which is shown in the figure 5. The range of work of this sensor is from $0 \mathrm{~Hz}$ to 100 $\mathrm{kHz}$ in the equivalent transduction from $0 \mathrm{~V}$ to $5 \mathrm{~V}$.

Figure 5. Vibration sensor [2].

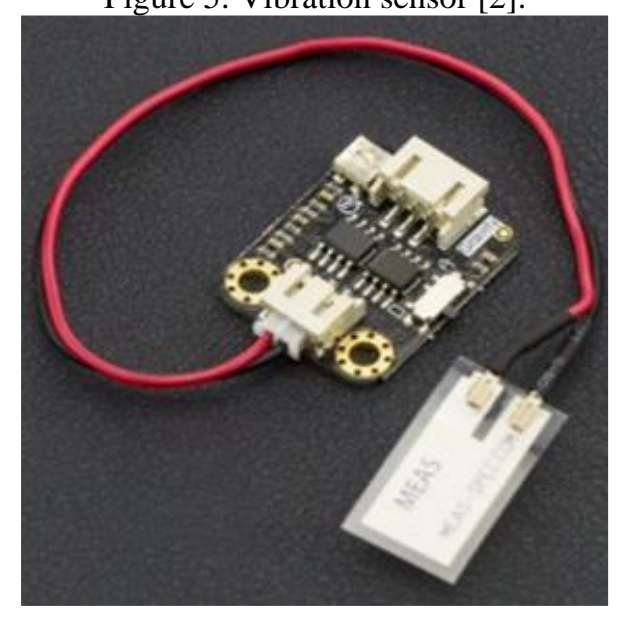

\subsection{TEMPERATURE SENSOR}

The vicuña wool washing process requires first soaking before being washed. For the washing process of the alpaca wool, it is necessary to immerse it into the water at a certain temperature (the requirement from 30 to 40 degrees Celsius). To analyze the range of variation, the temperature sensor $\mathrm{Lm}$ 35 (as it is showed in the figure 6) based on an integrated circuit, which has been used for an operating range of $10 \mathrm{mV}$ for each degree Celsius and an operating range within the requirements. 
Figure 6. Temperature sensor Lm 35 [3].

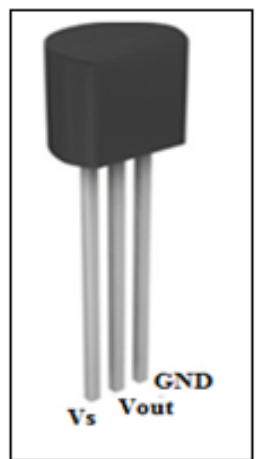

\subsection{TEMPERATURE CONTROLLER}

For the temperature controller, after the open-loop mathematical modelling study, a control algorithm of the temperature is carried out and the required frequency value is adjusted. The control signal is sent by the Arduino nano (as it is showed in the figure 7) that will execute a very simple, operational and functional control for this task.

Figura 7. Temperature Controller by Arduino nano.

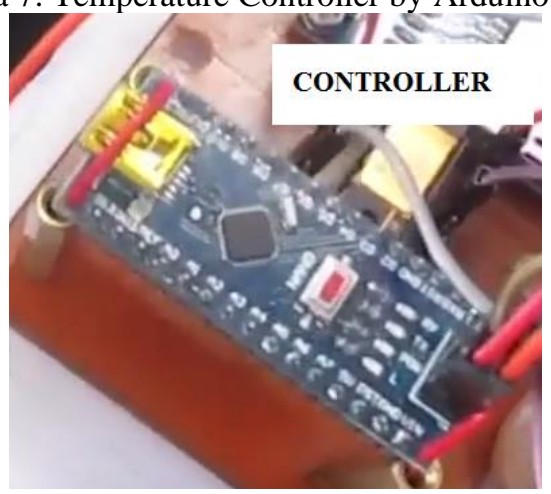

\subsection{ELECTRICAL RESISTANCE}

For the developed system, an electrical resistance is needed that through the Joule effect it can provide heat to the flow of the water that was used for the washing process (as the electrical resistance model that is showed in the figure 8).

Figura 8. Electrical resistance of $27 \mathrm{~W}$.

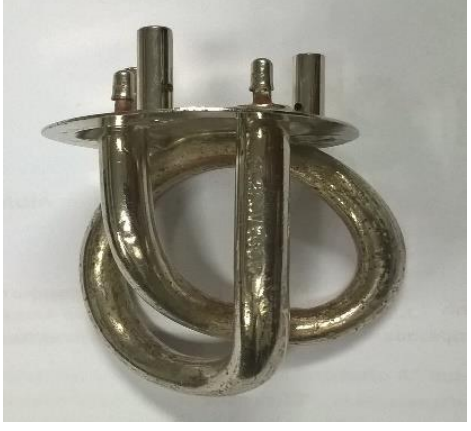




\section{SIMULATIONS}

For the simulations it was used the wave equation (Equation 1), where "P" is the sound source and where the vibration as the main source of the sound. "P" is a function that depends on position and time and its changes are characterized by the coefficients of the polynomial function. The Laplacian of this function allows generalizing for stationary states that tends to zero, also its evaluation at the distance "x" as a potential function convergent to "q", and " $\mathrm{C}$ " is the wave speed.

$$
\frac{1}{\mathrm{c}^{2}} \frac{\partial^{2}(\mathrm{P}(\mathrm{x}, \mathrm{t}))}{\partial \mathrm{t}^{2}}-\Delta(\mathrm{P}(\mathrm{x}, \mathrm{t}))=\mathrm{q}(\mathrm{x}, \mathrm{t})
$$

It is achieved the steady-state through the relationship between the source "P" and the measurement point "q", therefore the error "E" can be known. Likewise, this "E" error can be minimized by optimization techniques, such as Least Mean Square (LMS), and the design of simulation and experimentation algorithms.

Matrix analysis is necessary for a correct control of sound/vibration values throughout threedimensional space that is given by the equation 2 .

$$
\left(\begin{array}{cc}
\frac{1}{\mathrm{c}^{2}} & \frac{\partial^{2}\left(\mathrm{P}_{1}(\mathrm{x}, \mathrm{t})\right)}{\partial \mathrm{t}^{2}}-\Delta \mathrm{q}_{1}(x, t) \\
\frac{1}{\mathrm{c}^{2}} & \frac{\partial^{2}\left(\mathrm{P}_{2}(\mathrm{x}, \mathrm{t})\right)}{\partial \mathrm{t}^{2}}-\Delta \mathrm{q}_{2}(x, t) \\
\vdots \\
\frac{1}{\mathrm{c}^{2}} & \frac{\partial^{2}\left(\mathrm{P}_{n}(\mathrm{x}, \mathrm{t})\right)}{\partial \mathrm{t}^{2}}-\Delta \mathrm{q}_{n}(x, t)
\end{array}\right)=E(x, t)
$$

It should be noted that any of the matrix components, when they are tending to the stationary stage, they converge in the value that is indicated in the equation 3.

$$
\frac{P(S)}{\Delta \mathbf{u}(S)}=\frac{\mathbf{k}_{n}}{\tau s+1}
$$

By comparing any included component in equation 1 with the solution of equation 3 in steadystate, it is possible to know the theoretical/experimental coefficients, where the response time is " $\tau$ ", the proportional gain is "Kp" and the response to the electrical excitation signal is "U", which allows providing energy to the actuators.

The model of equation 3 is proposed as a stationary model, which algebraic operation in Laplace "S" is simple and prior to the temporary found solution. 
The flow diagram for the signal processing has been obtained in the figure 9. This diagram shows, by analyzing data that was taken from the sound, from $28 \mathrm{kHz}$ to $32 \mathrm{kHz}$, temperature and vibration sensors and with the described equation above are quite important to execute the designed algorithm.

Figure 9. Block diagram of the main algorithm.

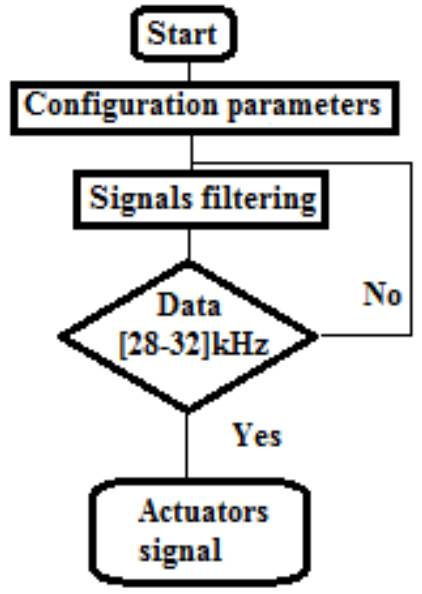

Through the obtained parameters, it is possible to characterize the geometric parameters that determine the decibels between actuators, when executing the algorithms as it is shown in the figure 10.

Figure 10. Simulation of the 2D wave transmition.

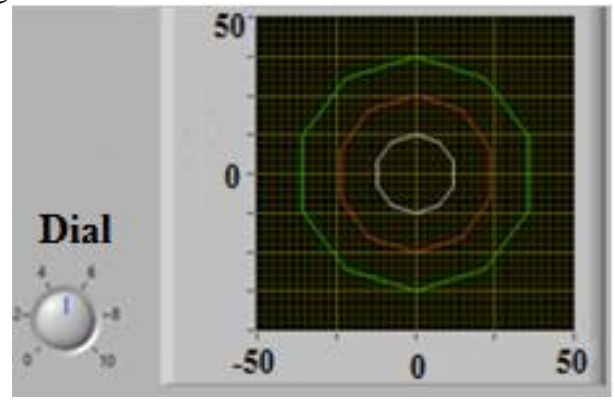

This calculation is obtained due to the speed of sound in water and the range of working frequencies from $10 \mathrm{kHz}$ to $30 \mathrm{kHz}$, which also are known and depicted by the simulation algorithm (the wave in 2 dimensions " $2 \mathrm{D}$ " in green, red and withe colour). To obtain these results allows the designed machine to work in optimal washing conditions and will allow obtaining the appropriate dimensions for the final model of the washing machine.

\section{EXPERIMENTAL ANALYSIS}

The experiments were made after the simulation results. The figure 11 shows the arrangement of the elements in $300 \mathrm{~mm}$ diameter tray containing water with a depth of $40 \mathrm{~mm}$. 
The equipment also has three actuators that emit a sinusoidal signal from $10 \mathrm{kHz}$ to $30 \mathrm{kHz}$ (signals generated in the Arduino controller) and the sound level meter that records the sound that was produced by the signal in the water.

Figure 11. Experimental setup.

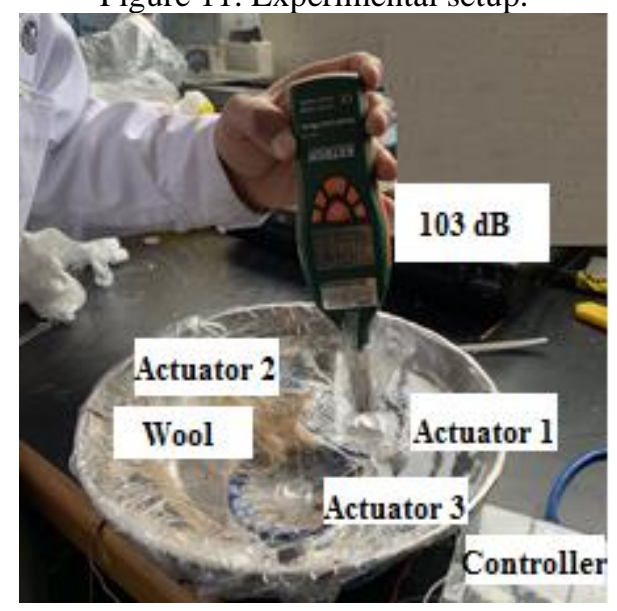

The figure 12 shows the three signals that are emitted from the three actuators. The signals, which have been monitored by the vibration/sound sensors, have a lag (dark green, light green and black curves) and these signals are not as "soft" as the emitted ones (violet, light blue and red curves).

Figure 12. Response in time domain.

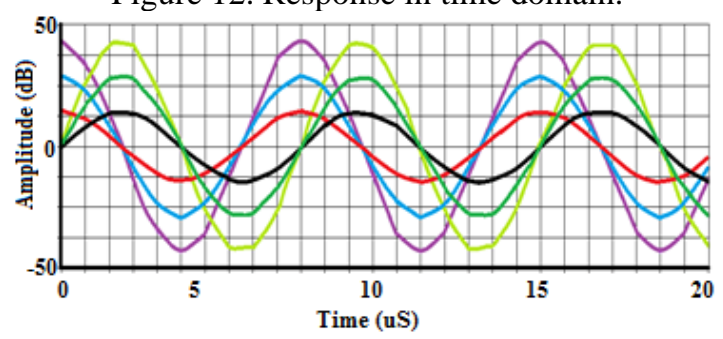

This phenomenon is due to the fact that data collection is subject to the effect of refraction and reflection. The frequency domain can give information that is not simple to achieve by time domain, such as the reflection and refraction of the vibration that was propagated by the ultrasonic source (the vibrator signal in blue colour) and they are getting answer through the water and walls of the washing machine (the vibrator answer in green, red and yellow colours). Hence, in the figure 13 is possible to identify the ideal cleaner frequency range value, which is from $28 \mathrm{~Hz}$ to $34 \mathrm{kHz}$. 
Figure 13. Response in frequency domain.

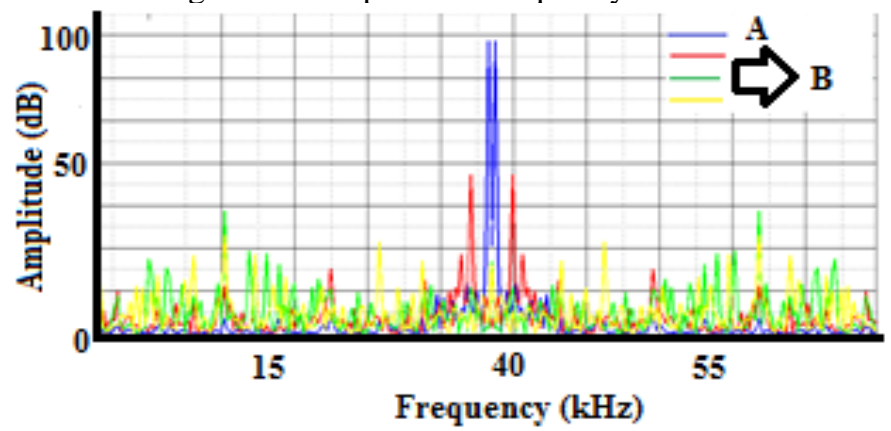

Finally, it was evaluated the 3D reconstruction algorithm due to analyze the performance of the optimization that was designed for the washing machine. The result of the cleaning can be obtained by different mechanisms of the chemical criterion. Nevertheless, many times chemical properties can not give enough information of solid residues that are stored in the fiber of the wool that is the reason, why the microscope in micro-scale was used. The raw wool (the figure 14A) and cleaned wool (the figure 14B) were compared by the $3 \mathrm{D}$ construction images, such as it is showed by the figure 14 too.

Figure 14. 2D images and their 3D reconstructions.

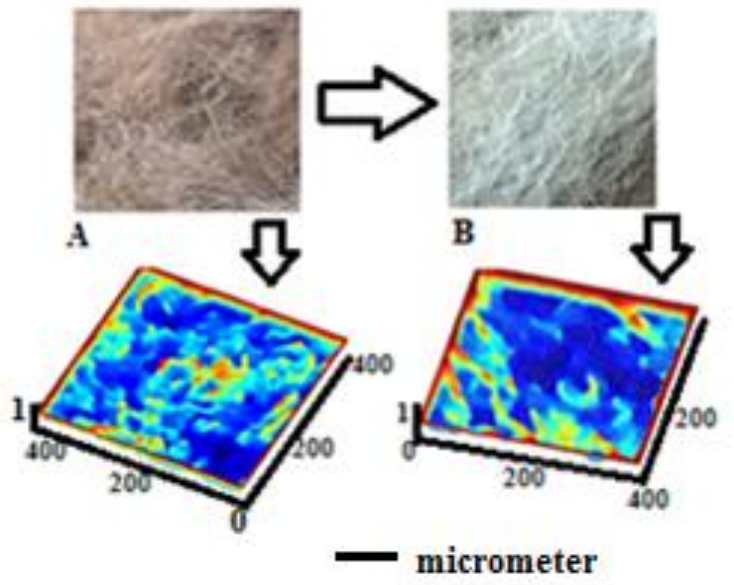

\section{CONCLUSIONS}

It was designed and proposed a smart ultrasonic system that makes it possible to wash vicuña wool without damaging it and reducing current energy consumption. The optimal range for frequency variation has been determined to be from $20 \mathrm{kHz}$ to $30 \mathrm{kHz}$.

It was necessary to use the method of $3 \mathrm{D}$ images reconstruction according to determine the evaluation and selection criterion of wool that is cleaned. Therefore, it was possible to identify cleaned wool after the ultrasonic washing.

The removing impurities system should be improved after the washing. 


\section{ACKNOWLEDGEMENTS}

The main author of this article J. Alan Calderón Ch., expresses:

-deep warm gratefulness to Mrs Aleksandra Ulianova de Calderón due to her total support for the development of this article according to find the explanation of the balance among human necessities and engineering solutions. Moreover the thankful because the vicuña photo proportionated in the figure 2 .

-it is expressed thankful to all co-authors due to their time and support for this research. Furthermore, by the academic cooperation between TU Ilmenau and PUCP that can open academic bridges between Peru and Germany.

-the special thankful to the leaders of the DGI ("Dirección de Gestión dela Investigación") researching office from PUCP, because of its financial support in this research through the financing FONCAI.

-the special gratitude to Prof. Hugo Medina, because of his teachings in Science Physics for many generations of engineers, he did and makes that physics laws could be so easy to get understanding of nature and current life, such as for this research. With a very good base of laws of nature, it was possible to obtain a fundamental to correlate advanced mathematics with the formalism that engineering applications always need.

-furthermore, it is declarated thankful to Mr. Broni Huamaní and Mr Leonardo Medina owing to their support in experimental tasks and simulation analysis. Both tasks are quite important in the evaluation of this article, because the critical analysis of the problematic needs the integrity of the mathematical modelling, from which is obtained the algorithm support for the simulations and prototype hardware design due to achieve the correlation between experiments and theory.

-the thankful to the financial support of the Concytec - World Bank Project "Improvement and Expansion of the Services of the National System of Science and Technology and Technological Innovation" 8682PE, through its executing unit ProCiencia [Contract Number 061-2018-FONDECYT-BM -IADT-MU] 


\section{REFERENCES}

[1] Adjustable frequency generator

https://www.bjultrasonic.com/shop/1500wultrasonic- generator/

19-06-2019.

[2] Vibrations sensor

https://www.didacticaselectronicas.com/index.php/sensores/vibracion/piezoelectrico,-piezo-electrico,zumbador,-vibrador-detail.

19-06-2019.

[3] Temperature sensor Lm35

https://www.alldatasheet.es/datasheetpdf/ pdf/8866/NSC/LM35.html.

19-06-2019.

[4] Arduino Controller Nano

https://www.arduino.cc/en/uploads/Main/ArduinoNanoManual23.

19-06-2019.

[5] F. John Fuchs, Ultrasonic cleaning, fundamental and applications, Blackstone Ultrasonics, Jamestown NY,

1995.

[6] Christopher James Hurren, A study into ultrasonic cleaning of wool, Deakin University, 2010.

[7] Mini Ultrasonic Cleaner Washing Device USB Machine Washer for travel home use.

https://www.e-crossmall.com/products/1982088282185

19-06-2019. 